\title{
A Practical Underwater Information Sensing System Based on Intermittent Chaos Under the Background of Lévy Noise
}

\section{Hanwen Zhang}

University of Electronic Science and Technology of China https://orcid.org/0000-0001-7568-5647

\section{Zhen Qin}

University of Electronic Science and Technology of China

Yichao Zhang

Southwest Jiaotong University

Dajiang Chen

University of Electronic Science and Technology of China

Ji Gen ( $\nabla$ jgeng@uestc.edu.cn )

University of Electronic Science and Technology of China

Hao Qin

University of Electronic Science and Technology of China

\section{Research Article}

Keywords: Underwater information sensing, Lévy Noise, Chaotic oscillator

Posted Date: January 10th, 2022

DOI: https://doi.org/10.21203/rs.3.rs-1196606/v1

License: (c) (i) This work is licensed under a Creative Commons Attribution 4.0 International License.

Read Full License 
Correspondence:

jgeng@uestc.edu.cn

${ }^{3}$ Network and Data Security Key

Laboratory of Sichuan Province,

Chengdu, China

Full list of author information is

available at the end of the article

\begin{abstract}
The Gaussian noise model has been chosen for underwater information sensing tasks under substantial interference for most of the research at present. However, it often contains a strong impact and does not conform to the Gaussian distribution. In this paper, a practical underwater information sensing system is proposed based on intermittent chaos under the background of Lévy noise. In this system, a novel Lévy noise model is presented to describe the underwater natural environment interference and estimate its parameters, which can better describe the impact characteristics of the underwater environment. Then an underwater environment sensing method of dual-coupled intermittent chaotic Duffing oscillator is improved by using the variable step-size method and scale transformation. The simulation results show that the method can sense weak signals and estimate their frequencies under the background of strong Lévy noise, and the estimation error is as low as $0.03 \%$. Compared with the intermittent chaos of the single Duffing oscillator and the intermittent chaotic Duffing of double coupling, the minimum SNR ratio threshold has been reduced by $11.5 \mathrm{~dB}$ and $6.9 \mathrm{~dB}$, respectively, and the computational cost significantly reduced, and the sensing efficiency is significantly improved.
\end{abstract}

Keywords: Underwater information sensing; Lévy noise; Chaotic oscillator

\section{Introduction}

With the progress of modern technology, e.g., Internet of things (IOT) [1, 2, 3], and artificial intelligence (AI) $[4,5]$, people began to develop and utilize marine resources $[6,7]$. People at home and abroad have conducted extensive research on underwater target technology to sense the underwater environment and develop underwater resources [8,9]. Most of the current studies choose the Gaussian noise model for discussion. $[10,11]$ This is because non-Gaussian noise does not have Markov characteristics and is very difficult to deal with in the research of weak signal sensing $[12,13,14]$. However, the noise interference of underwater environment often contains intense pulses and does not conform to Gaussian distribution [15, 16, 17]. Lévy distribution is a generalized form of Gaussian distribution. It is a typical nonGaussian noise with a long tail, discontinuous jump, and infinite separability. It can maintain the natural noise process's generation mechanism and propagation conditions. The Lévy noise model established by Lévy distribution can describe many symmetrical or asymmetric noises with different impulsivity by controlling the selection of different parameters. [18, 19]. It has more substantial applicability and 
great significance to describe the influence characteristics of underwater environmental noise interference [20] . In addition, in the current research on the sensing of underwater unknown frequency signals [21, 20], there are problems that the sensing method can not fully estimate the unknown frequency signal parameters [22, 23, 24], and the signal-to-noise ratio threshold is too high [25, 26, 27, 28, 29].

This paper proposes an underwater position sensing method that is more suitable for weak signals in a complex ocean environment to solve the above problems. The main contributions of this paper are as follows:

- Lévy noise model is proposed to describe the underwater natural environment, and an analysis and estimation method is provided to select better parameters.

- Aiming at the sensing problem of weak underwater signal with unknown frequency, and improved dual-coupled Duffing oscillator signal sensing method based on scale change and variable step size is proposed. This method has better resistance to strong noise, better adaptability to all kinds of impact noise, higher sensing efficiency, and more intuitive sensing results, and with a lower threshold.

- An underwater weak signal sensing system with unknown frequency is designed and established. The system can better sense multi-frequency signals under Lévy noise model. The feasibility and superiority of the sensing system established in this paper are verified by using the actual underwater acoustic data.

\section{Related Work}

\subsection{Lévy noise model}

The Lévy process was proposed by the French mathematician Paul Lévy to study of the generalized central limit theorem. It is a random process with independent and fixed increments, indicating that the movement of a point and its continuous displacement are random [30]. The difference between two disjoint time intervals displacement is independent. The displacement and displacement in different time intervals of the same length have the same probability distribution. It can be regarded as a continuous-time simulation of random walk. Lévy noise, also called alpha noise, obeys the theory of stable alpha distribution. The only distribution satisfies the generalized central limit theorem,and a square law attenuates its tailing. The expression of the characteristic function of Lévy noise [31], is as follows:

$$
\begin{aligned}
& \log \varphi(t)= \begin{cases}-\sigma^{\alpha}|t|\left\{1-i \beta \operatorname{sign}(t) \tan \left(\frac{\pi \alpha}{2}\right)\right\}+i \mu t, \quad \alpha \neq 1 \\
-\sigma|t|\left\{1+i \beta \operatorname{sign}(t) \frac{\pi}{2} \log (|t|)\right\}+i \mu t, \quad \alpha=1\end{cases} \\
& \begin{cases}X=S_{\alpha, \beta} \frac{\sin \left(\alpha\left(V+B_{\alpha, \beta}\right)\right)}{(\cos V)^{\frac{1}{\alpha}}}\left(\frac{\cos \left(V-\alpha\left(V+B_{\alpha, \beta}\right)\right)}{W}\right)^{\frac{1-\alpha}{\alpha}}, & \alpha \neq 1 \\
X=\frac{2}{\pi}\left[\left(\frac{\pi}{2}+\beta V\right) \tan V-\beta \log \left(\frac{W \cos V}{\frac{\pi}{2}+\beta V}\right)\right], & \alpha=1\end{cases}
\end{aligned}
$$

In (1), $\alpha \in(0,2]$ is the characteristic index, which determines the decay rate of the distribution tail. When $\alpha=1$, it is Cauchy distribution. When $\alpha=2$, It is the Gaussian distribution, and the mean value is $\mu$, the variance is $2 \sigma^{2}$. When 
$\alpha \neq 2$, the mean value is $\mu$, but the variance does not exist. $\beta \in[-1,1]$ is the skew parameter. When $\beta=0$, the graph is symmetrical, and when $\beta$ is a positive number, The graph tilts to the right, on the contrary, the graph tilts to the left, $\sigma>0$ is the scale parameter, which deter- mines the degree of dispersion of the distribution concerning $\mu$ and $\mu \in R$ is the position parameter. The left and right translation can be achieved by adjusting the value of $\mu$. Rfal-Weron proved the expression of Lévy distribution random variable. In (2), V obeys the uniform distribution in the interval $(-2 \pi, 2 \pi), W$ follows the exponential distribution with the mean value 1 , $S_{\alpha \beta}$ and $B_{\alpha \beta}$ is as follows:

$$
\begin{aligned}
& S_{\alpha, \beta}=\left[1+\beta^{2} \tan ^{2} \frac{\pi \alpha}{2}\right]^{1 / 2 \pi} \\
& B_{\alpha, \beta}=\frac{\arctan \left(\beta \tan \frac{\pi \alpha}{2}\right)}{2}
\end{aligned}
$$

Under the conditions of $\beta=0, \sigma=1$ and $\mu=0$, the Lévy distributions corresponding to different $\alpha$ feature indices are shown in Figure 1:
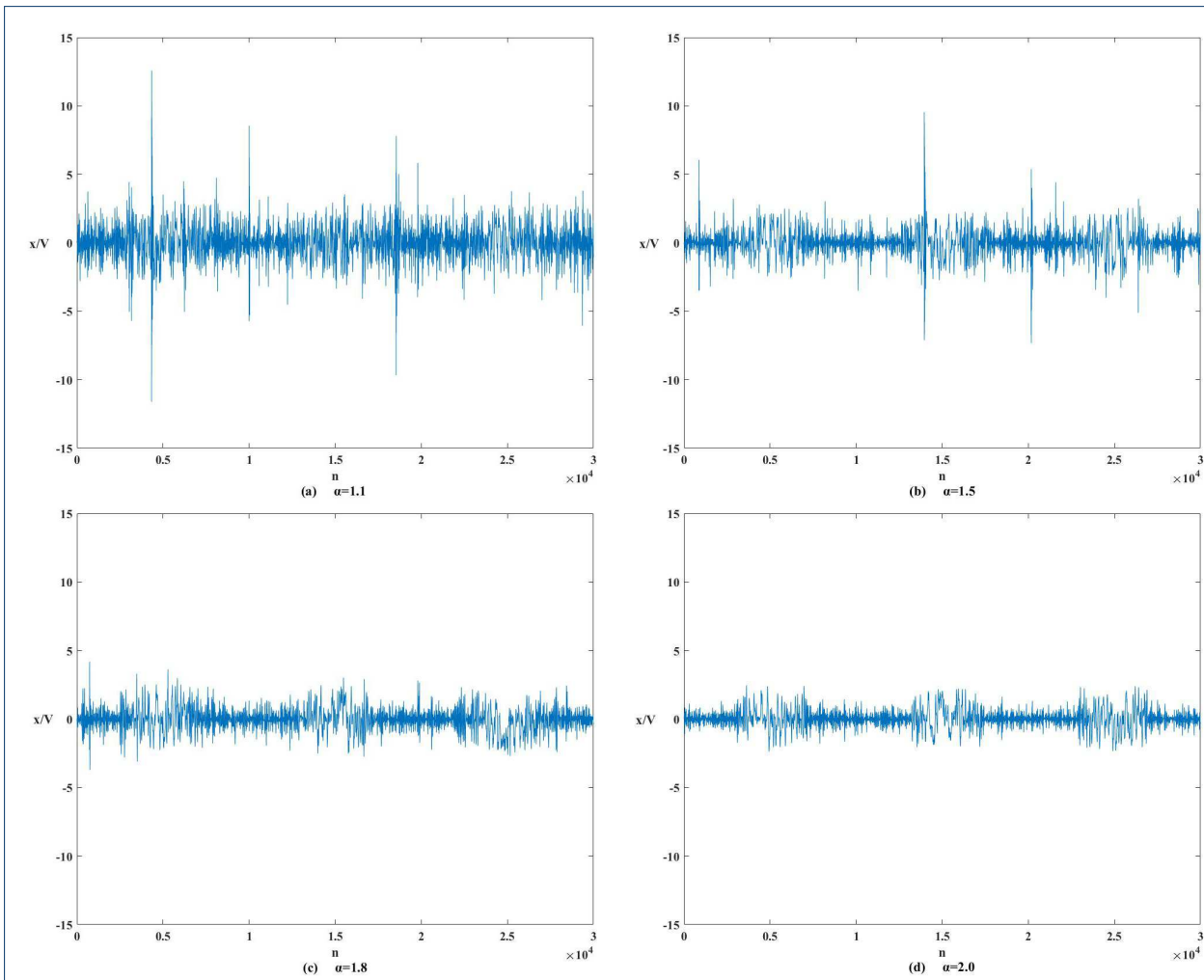

Figure 1 Time-domain distribution map of live noise corresponding to different feature index $\alpha$

It can be seen from Figure 1 that the smaller the value $\alpha$, the stronger the impact of noise interference. When $\alpha=2$, the noise interference has almost no impact. At this time, the Lévy noise degenerates into white noise. 


\subsection{Traditional Chaotic oscillator sensing system}

The nonlinear dynamic system described by Duffing equation exhibits rich nonlinear dynamic characteristics, including complex oscillation dynamics, bifurcation, and chaos. The specific form of a single Duffing oscillator equation is:

$$
x \ddot{(} t)+k x \dot{(t})-x(t)+x^{3}(t)=F \cos (\omega t)
$$

Where $x(t)$ is the chaotic system variable; $k$ is the damping ratio; $t$ is the time variable; $F \cos (\omega t)$ is the periodic driving force, where $F$ is the amplitude of the periodic driving force, and $\omega$ is the angular frequency of the periodic driving force; $-x(t)+x^{3}(t)$ is the nonlinear restoring force. When $k$ is fixed, the system's state changes regularly with the amplitude of the driving force $F \cos (\omega t)$. Measured by the simulation experiment, when $F$ increases to 0.82673 , the system enters the critical state of large-scale periodic motion. At this time, the magnitude of the driving force 0.82673 is the critical threshold for the transition from chaos to a periodic state. The signal sensing method using a single Duffing vibrator can effectively sense the signal, but the immunity to noise interference is low. If the interference is too strong, the phase trajectory will remain in a chaotic state, resulting in an illusion that the signal is not sensed, and misjudgment occurs.

\section{Methods}

3.1. Lévy noise model describes underwater natural environment interference

The Fokker-Plank equation corresponding to equation (1) is:

$$
\frac{\partial_{p}(s, t)}{\partial t}=\left[\frac{\partial}{\partial x} A(x)+\frac{\partial^{2}}{\partial x} B(x)\right] \rho(s, t)
$$

Where $A(x)=a x-b x^{3}+S(x), B(x)=D$. Since equation (7) is a transcendental equation, it cannot be solved directly, but the approximate number of equation (6) can be calculated using the finite difference method. Set the system parameters $a=0.6, b=0.3, A=0.3, f=0.005$, noise parameters $\alpha=1.5, \beta=0, \sigma=1, \mu=0$, the probability distribution curve of particle density is shown in Figure 2.

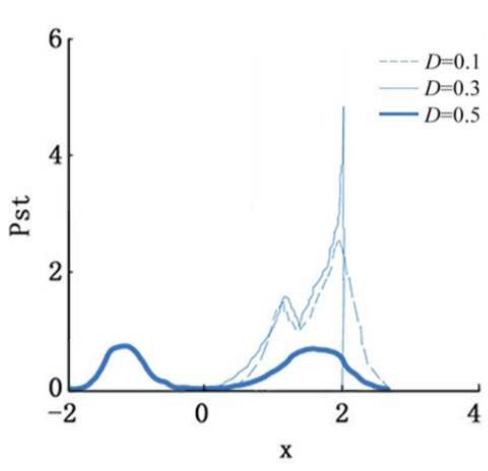

Figure 2 The particle probability distribution of the system output under the excitation of different noise intensity $D$ 
When the Lévy noise intensity is 0 , the probability distribution of particle density is only affected by the signal. When the noise intensity $D$ increases from 0.1 to 0.3 , the particle density probability indicates that the particles are mainly concentrated on the side of point 0 . When the noise intensity $D$ reaches 0.5 , the particles are unevenly distributed on both sides of 0 . From the micro-particle motion law, when the noise intensity $D$ is 0.3 , the external noise excitation intensity is not enough. The energy obtained by the particle is not enough to make it pass through the potential barrier and can only move left and right in the potential well, so the probability of the particle is limited. The distribution is concentrated on side 0 . The resultant force of signal tension and noise interference received by particles at 0 determines whether they are distributed in the left potential well or the right potential well. When the noise intensity is 0.5 , solid noise will excite particles. They will gain enough energy to pass through the barrier from one potential well to another. At this time, the particle transfer between potential wells will be affected by noise. Therefore, the particle distribution on both sides of point 0 will never be symmetrical. From Figure 2, we can get a message. When the characteristic index is constant, the larger the external excitation energy obtained by the particle, the more it can move through the potential barrier to another potential well. That is, the greater the noise intensity, the more particles between the potential wells. The higher the crossover frequency, so we need to pay special attention to the characteristic index when selecting the parameters of the Lévy noise model $\alpha$ And noise intensity $D$ [32].

In this regard, this paper quotes the method of literature [33], to estimate the characteristic index $\alpha$ and the noise intensity $D$ of the Lévy noise model:

$$
E\left(|X|^{\rho}\right)=\frac{\rho l g \alpha}{\alpha \lg D} C(\rho, \alpha)
$$

Where $E\left(|X|^{\rho}\right)$ is the fractional low-order moment, and $\rho$ is the order, $C(\rho, \alpha)=$ $\frac{2^{\rho+1} \tau\left(\frac{\rho+1}{2}\right) \tau\left(\frac{-\rho}{\alpha}\right)}{\alpha \sqrt{\pi} \tau\left(\frac{-\rho}{2}\right)},-1<\rho<\alpha \leq 2$. Let $Y=l g|X| E(Y)<+\infty$, the moment generating function is:

$$
E\left(|X|^{\rho}\right)=\lim _{\rho \rightarrow 0} \frac{d^{q}}{d \rho^{q}} E\left(|X|^{\rho}\right), q \in N^{*}
$$

Since $Y$ is only related to $\alpha$ except for the first moment, the first two finite logarithmic moments are listed as:

$$
\left\{\begin{array}{l}
G_{1}=E\left(|X|^{\rho}\right)=\Phi_{0}\left(1-\frac{1}{\alpha}\right)+\lg \left|\frac{\lg \alpha}{\lg D \cos k}\right|^{\frac{1}{\alpha}} \\
G_{2}=E\left[(l g|X|-E(Y))^{2}\right]=\Phi_{1}\left(\frac{1}{2}+\frac{1}{\alpha^{2}}\right)-\left(\frac{k}{\alpha}\right)^{2} \\
\Phi_{0}=\frac{d l g \tau(t)}{d t} \\
\Phi_{1}=\frac{d^{2} l g \tau(t)}{d t^{2}}
\end{array}\right.
$$

Let $t=1$, then $\Phi_{0}=-0.5772$ and $\Phi_{1}=1.6449$ are obtained. The estimated value can be obtained from the above formula: 


$$
\left\{\begin{aligned}
\widehat{\alpha} & =\left(\frac{G_{2}}{\Phi_{1}}-\frac{1}{2}\right)^{-\frac{1}{2}} \\
\widehat{D} & =e^{\left(\Phi_{0}-G_{1}\right) \widehat{\alpha}-1} \frac{\lg \widehat{\alpha}}{\cos \widehat{k}} \\
|\widehat{k}| & =\left[\left(\frac{\widehat{\alpha}^{2}}{2}-1\right) \Phi_{1}-G_{1} \widehat{\alpha}^{2}\right]^{\frac{1}{2}}
\end{aligned}\right.
$$

To test the effectiveness of the estimation method, the Chambers-Mallows-Stuck (CMS) method is used to generate the Lévy noise with $\alpha=1.5, D=1$, and the parameters of $\alpha, D$ are estimated. The estimated results are shown in the figure below:

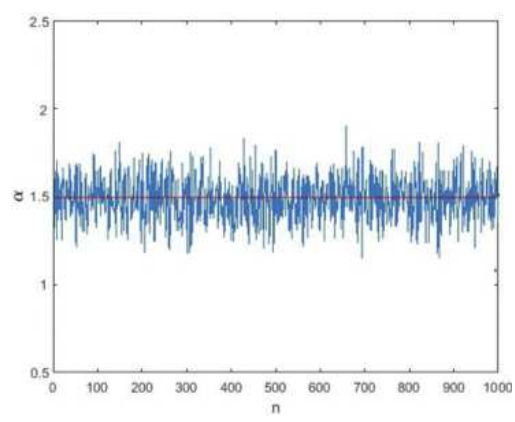

Figure 3 Estimation of characteristic index $\alpha$

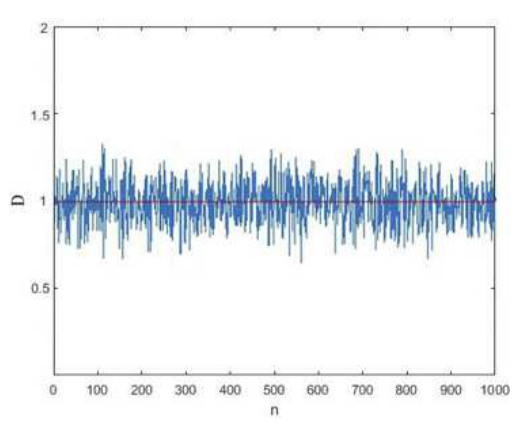

Figure 4 Estimation of noise intensity $D$

Where $n$ represents the number of estimates, figure 4 and figure 5 show that values of $\alpha$ and $D$ are obtained respectively as 1.5026 and 1.1664 , which prove that the method can estimate the parameters of interference noise in the actual underwater information sensing environment.

\subsection{Improved signal sensing method of dual-coupled Duffing oscillator}

A. Intermittent chaos of Duffing oscillator

The intermittent chaos of the Duffing oscillator uses the phase change of the Duffing oscillator to sense weak signals $[34,35,36]$. If there is a small frequency difference between the signal under test and the built-in driving force of the system, the 
system will produce intermittent chaos phenomenon in which the chaotic state and the periodic state alternately appear. This method uses the immunity of Duffing oscillator to noise and the sensitivity of the critical chaotic state of Duffing oscillator to small disturbances to realize the sensing of weak signals [37].

1. Intermittent chaos of a single Duffing oscillator Add $f(t)$ as the signal to be sensed into the single Duffing vibrator sensing system, then the state equation is:

$$
\left\{\begin{array}{l}
\dot{x}=\omega y \\
\dot{y}=\omega\left[-0.5 y+x-x^{3}+\gamma \cos (\omega t)+f(t)\right]
\end{array}\right.
$$

Where $f(t)=A \cos (\dot{\omega} t+\varphi), \varphi$ is the initial phase of the signal to be sensed, $\gamma$ is the critical chaos threshold, $\dot{\omega}=\omega+\Delta \omega, \Delta \omega$ is the frequency difference between the built-in driving force of the system and the signal to be sensed. The overall driving force of the system is:

$$
L(t)=\gamma \cos (\omega t)+A \cos (\dot{\omega} t+\varphi)=P(t) \cos (\omega t+\phi(t))
$$

In the formula $(12), P(t)$ represents the polarization force amplitude:

$$
P(t)=\sqrt{\gamma^{2}+2 \gamma A \cos (\Delta \omega t+\phi)+A^{2}}, \quad A \ll \gamma
$$

When $\left|\frac{\Delta \omega}{\omega}\right| \leq 0.03$, the output sequence of the system is shown in Figure 5(a), and it is observed that Figure 5 is an obvious intermittent chaotic state. When $\left|\frac{\Delta \omega}{\omega}\right|>0.03$, the timing diagram of system output cannot be judged as intermittent chaotic state, as shown in Figure 5(b).

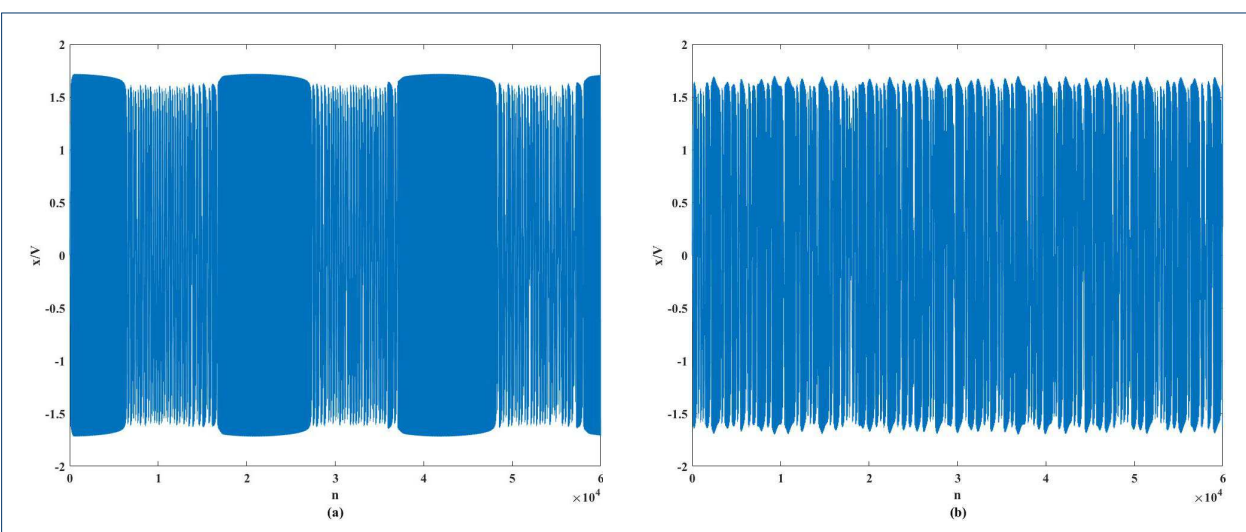

Figure 5 The output sequence of the single Duffing oscillator system

2. Intermittent chaos of dual-coupled Duffing oscillator Add $f(t)$ as the signal to be sensed into the dual-coupled Duffing oscillator sensing system, then the state equation is: 


$$
\left\{\begin{array}{l}
x \ddot{(t} t)+k x \dot{(t})-x(t)+x^{3}(t)+d(y(t)-x(t))=\gamma F \cos (\omega t) \\
\ddot{y}(t)+k y \dot{(t)}-y(t)+y^{3}(t)+d(x(t)-y(t))=\xi \gamma F \cos (\omega t)+f(t)
\end{array}\right.
$$

Where $f(t)=A \cos (\dot{\omega} t+\varphi), \dot{\omega}=\omega+\Delta \omega$. When $\left|\frac{\Delta \omega}{\omega}\right| \leq 0.08$, the output sequence of the system is shown in Figure 6(a), which is an obvious intermittent chaotic state. When $\left|\frac{\Delta \omega}{\omega}\right|>0.08$, the timing diagram of the system output cannot be judged as an intermittent chaotic state, as shown in Figure 6(b).
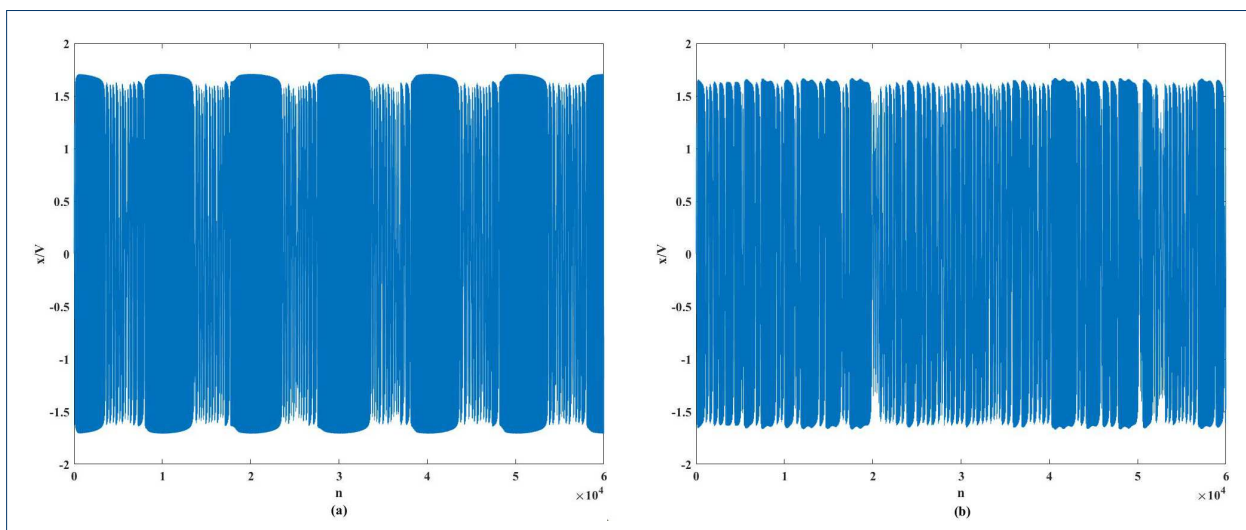

Figure 6 The output sequence of the dual-coupled Duffing oscillator system

\section{B. Improved signal sensing method of intermittent chaotic dual-coupled Duffing oscillator}

Although the signal sensing method of the intermittent chaotic dual-coupled Duffing oscillator improves the speed and accuracy of a certain SNR ratio and threshold solution, it still cannot solve the problem of estimating the unknown frequency signal parameters and the high SNR ratio threshold [38]. In this paper, combining the theoretical knowledge of scale transformation and variable step-size method, an improved signal sensing method based on the scale transformation of the variable step size intermittent chaotic differential dual-coupled Duffing oscillator is proposed. The estimated result of the sensed unknown signal frequency is more accurate, the SNR ratio threshold is lower, and the calculation overhead is smaller.

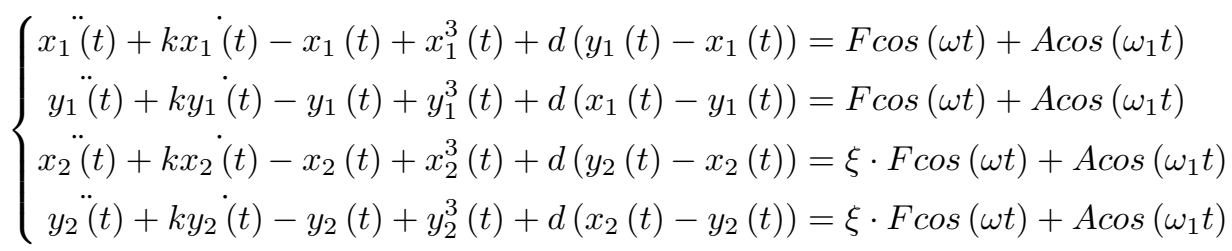

Where $\xi$ is the influencing parameter of the chaotic oscillator, the other parameters of the two pairs of Duffing oscillators are exactly the same. By generating the 
differential timing diagrams $x_{1}(t)-x_{2}(t)$ of the two pairs of Duffing oscillators to observe whether the signal has been sensed. The timing diagram should be a regular timing diagram, the change of $\xi$ will not affect the waveform, only the magnitude of the phase difference. When $\xi=1$ the phase difference disappears and the two pairs of oscillators are exactly the same. At this time, they will degenerate into ordinary dual-coupled Duffing oscillators. In this paper, through a lot of experiments, the final choice of $\xi=1.001$ has obtained a more intuitive effect.

In this paper, the fourth-order Runge-Kutta method is used to numerically analyze the Duffing vibrator sensing system. Through calculation, when the frequency difference range of intermittent chaos is $\left|\frac{\Delta \omega}{\omega}\right|<0.09$ the system is in the state of intermittent chaos. Comparing the intermittent chaotic frequency difference range of the three methods, we can get that the method proposed in this paper can better detect the step size, which makes the use of the variable step size method more meaningful and can more easily realize the signal sensing of unknown frequencies. The intermittent chaotic sequence diagram of the method proposed in this paper is shown in the Figure7:

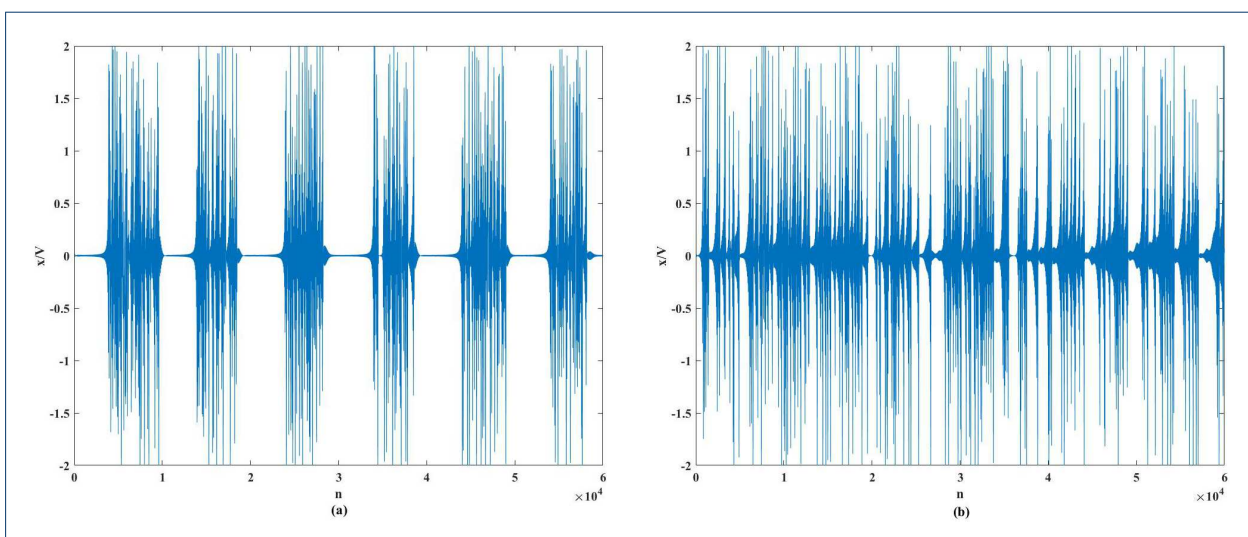

Figure 7 The output sequence of improved method

Next, apply variable step size on the basis of the above. Change the solution step length of the solution process, and convert the signal into the corresponding discrete sequence of the built-in driving force of the sensing system. By observing the system output $x_{1}(t)$ timing diagram and $x_{1}(t)-x_{2}(t)$ differential timing diagram to determine whether the sensing is successful. Let the angular frequency of the built-in signal of the sensing system be $\omega$ For the signal to be measured with an angular frequency of $\omega_{1}$, the conventional system solution step is the interval of the built-in driving force sequence of the sensing system, the sequence interval of the signal to be sensed is $T_{s}\left(T_{s}=\frac{1}{f_{s}}, f_{s}\right.$ is the sampling rate of the signal). The sensing result is only related to the sampling rate and has nothing to do with the step length. Therefore, the built-in driving force sequence interval of the sensing system can be changed until the system is adjusted to an intermittent chaotic state, thereby completing signal sensing.

The total strategy term of equation (15) is $F \cos (\omega t)+A \cos \left(\omega_{1} t\right)$ and $\xi F \cos (\omega t)+$ $A \cos \left(\omega_{1} t\right)$. When the system is in an intermittent chaotic state, set the built-in driving force sequence interval to $\frac{\dot{\omega}}{s \omega f_{s}}, \dot{\omega}=\omega+\Delta \omega, s \in(0.91,1.09)$. This is equivalent 
to changing the signal to be measured by $\frac{\Delta \omega}{\omega}$ times on the time axis, so that the relative angular frequency with the built-in signal becomes $1: 1$. Under this condition, the influence of the signal under test on the output phase of the system is equivalent to a signal with the same frequency as the built-in driving force. This means that the improved algorithm for the built-in signal angular frequency $\omega$ can transform the step size to make the signal under test whose frequency is not in the range of $(0.91,1.09) \omega$, and it can also make the sensing system appear intermittent chaotic. When the variable step size method is applied, the two strategy items are discretized as follows:

$$
\left\{\begin{array}{l}
L_{n_{1}}=F \cos \left(\frac{n \dot{\omega}}{s f_{s}}\right)+A \cos \left(\frac{n \dot{\omega}}{f_{s}}\right) \\
L_{n_{2}}=\xi \cdot F \cos \left(\frac{n \dot{\omega}}{s f_{s}}\right)+A \cos \left(\frac{n \dot{\omega}}{f_{s}}\right)
\end{array}\right.
$$

At this time, reconstruct a new chaotic oscillator sequence with length $n$ and a common ratio $\left|\frac{\omega+\Delta \omega}{\omega}\right|$ satisfying $\left|\frac{\Delta \omega}{\omega}\right|<0.09$. The sequence is expressed as:

$$
\omega_{1}=1, \quad \omega_{2}=\left|\frac{\omega+\Delta \omega}{\omega}\right| \omega_{1}, \quad \omega_{3}=\left|\frac{\omega+\Delta \omega}{\omega}\right| \omega_{2}, \quad \ldots, \quad \omega_{n}=\left|\frac{\omega+\Delta \omega}{\omega}\right| \omega_{n-1}
$$

Bring the oscillator array into (12) to find the critical chaotic state frequency difference threshold of the sensing system under the current chaotic oscillator array, and adjust the built-in driving force sequence interval $a_{n}=2 \pi \frac{s^{n}}{s \omega f_{s}} \quad,(n=1,2, \ldots, N)$ and observe the system output timing diagram, if the frequency difference between the built-in driving force and the signal meets the standard of intermittent chaos, the sensing system will appear intermittent chaos. The specific steps are shown in the figure below:

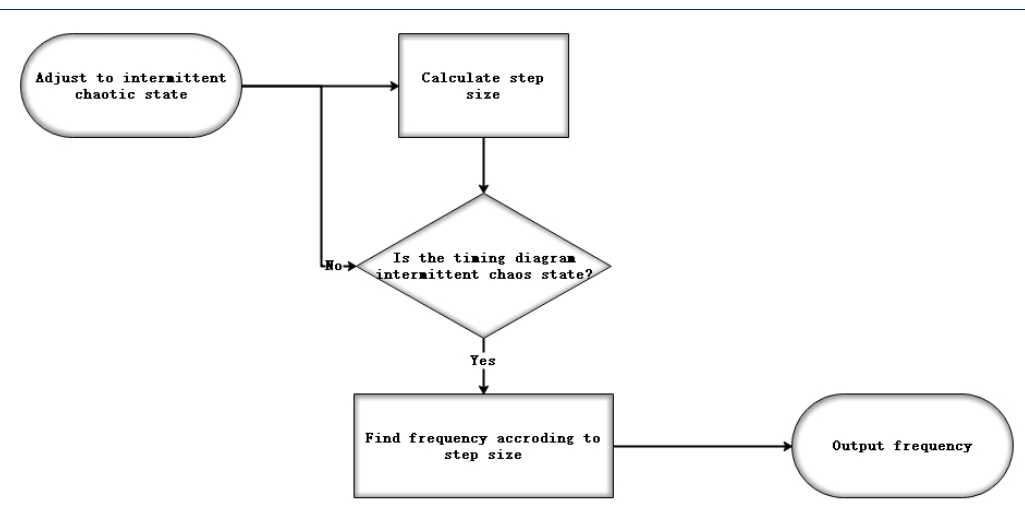

Figure 8 The specific steps of the chaotic sensing system

When two adjacent built-in driving force sequence intervals are in an intermittent chaotic state, the existence and frequency range of the signal to be measured can be determined. When the noise intensity is too high, the state of the Duffing oscillator's sensing system will be greatly affected by the noise, which will cause the system to enter the wrong chaotic state, and make the weak signal sensing wrong, 
as shown in Figure 9.

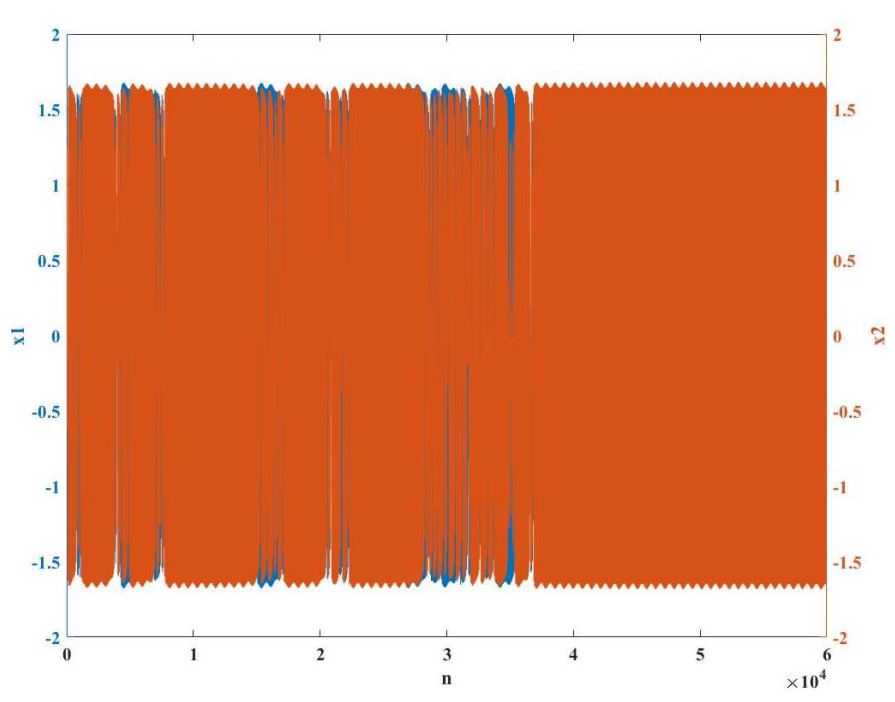

Figure 9 The the wrong chaotic state of the chaotic sensing system

Therefore, this paper adopts the method of calculating the standard deviation $\sigma_{s}$ of the input signal, and automatically scales the signal under test superimposed with noise through the standard deviation. First, collect a piece of input signal $f(t)$ of length $N$, calculate the standard deviation of the sampling sequence $\sigma_{s}^{2}=E x^{2}-E^{2} x$, and set a target standard deviation value $\sigma_{s d}$ based on this. Then calculate the standard deviation of the collected signals once and construct the scale conversion factor $k=\frac{\sigma_{s d}}{\sigma_{s}}$. If $k<1$, multiply the sampled signal by $k$ to obtain the transformed sample sequence, and then send it to the signal sensing system. Let the strategy term after the equation be $U(t)=A \frac{\sigma_{s d}}{\sigma_{s}} \cos \left(\omega_{1} t\right)$. The system expression using scale transformation is as follows:

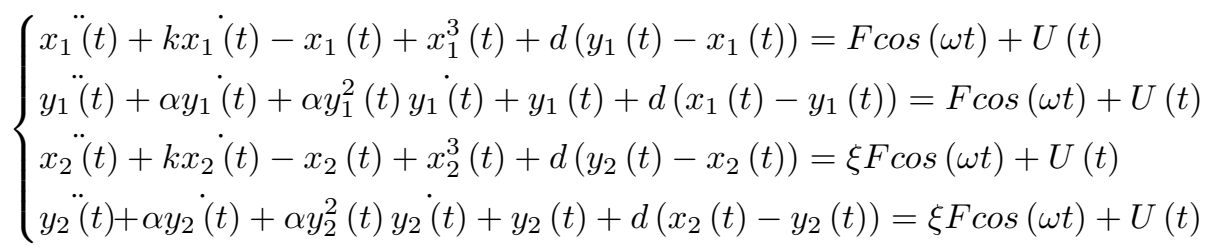

The parameters shown in Figure 10 are added to the sensing system after the scale transformation is introduced, and the output state is shown in Figure 11. It can be seen that the anti-interference ability of the sensing system against noise is significantly improved after the scale conversion is added. The detection SNR ratio threshold is obviously reduced. 


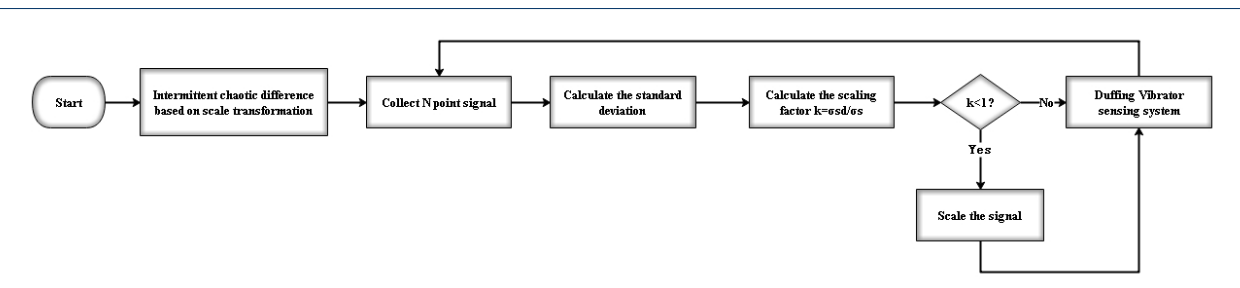

Figure 10 The scale transformation flow chart

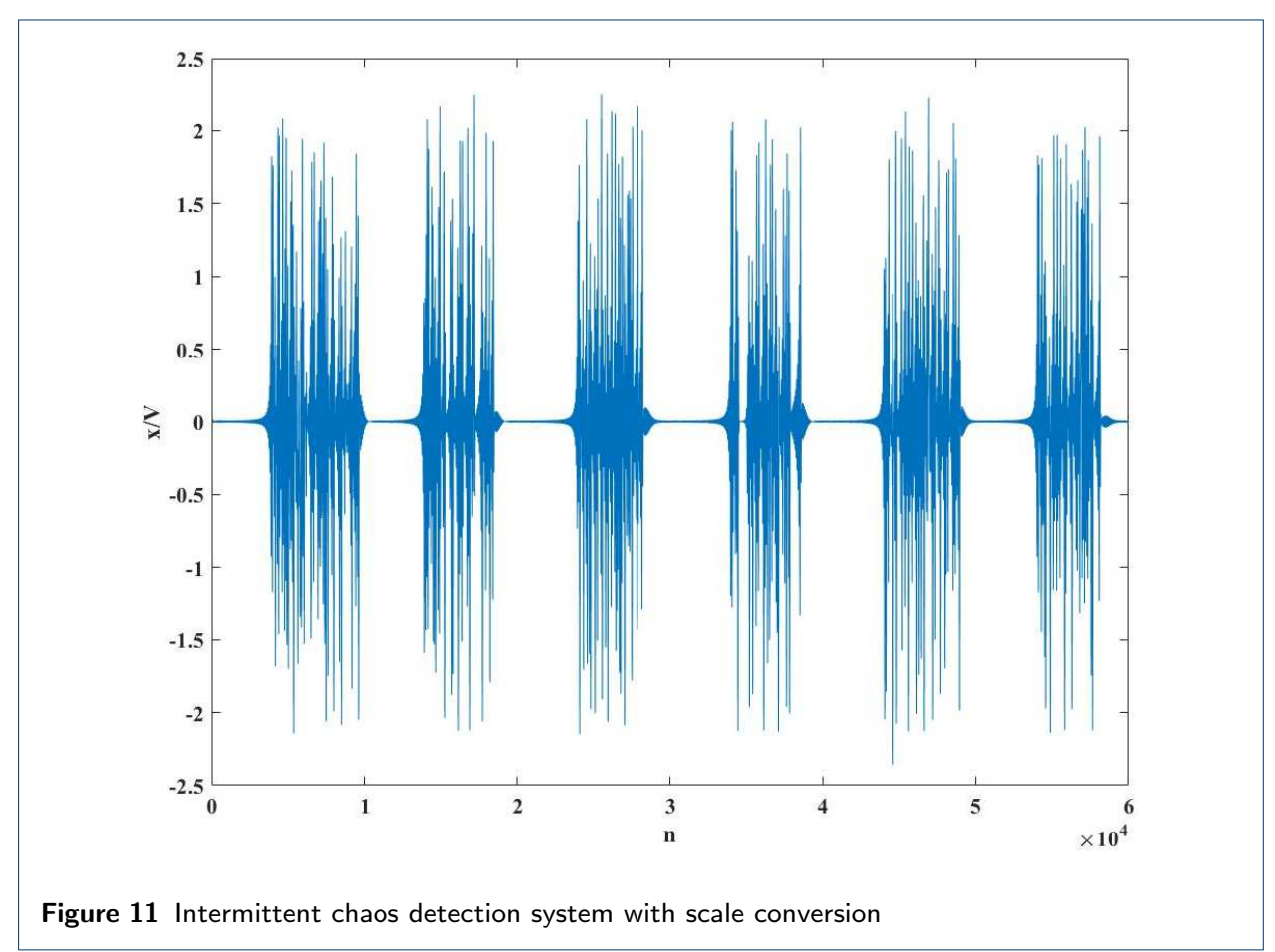

\section{Results and Discussion}

In order to describe the actual marine environment more effectively, this paper first uses the Chambers-Mallows-Stuck (CMS) method to generate Lévy noise with $D=1$, The values of $\alpha$ are 1.5, 1.8 and 2. Under the interference of Lévy noise model, an intermittent chaotic signal sensing system based on the improved scale transform intermittent chaotic variable step size dual-coupled Duffing oscillator is established. The simulation proves the efficiency and superiority of the sensing system. Finally, in order to verify the effectiveness of the actual application of the method in this paper, the underwater acoustic data of a reservoir in Sichuan is used for the experiment.

\section{A. The influence of system coupling coefficient on system performance} Under normal circumstances, the higher the coupling strength of the system, the stronger the synchronization between the oscillators. $D$ in formula (18) represents the coupling coefficient. In order to further compare the noise immunity and stability of the coupling system corresponding to different coupling coefficients, the concept of standard deviation is introduced: 


$$
\sigma=\sqrt{\frac{1}{N-1} \sum_{t=1}^{N}\left[x_{n}(t)-x_{0}(t)\right]^{2}}
$$

Calculate the standard deviation of different coupling coefficients $d$ under different noise backgrounds, as shown in Table 1 and Table 2:

Table 1 The standard deviation of different coupling coefficients $d$ under different noise backgrounds $(D=1)$

\begin{tabular}{cccc}
\hline$d / \alpha$ & $\alpha=1.5$ & $\alpha=1.8$ & $\alpha=2$ \\
\hline$d=0$ & 1.906 & 1.057 & 1.054 \\
$d=0.1$ & 1.095 & 1.039 & 1.034 \\
$d=0.2$ & 1.039 & 0.972 & 0.96 \\
$d=0.3$ & 1.056 & 0.988 & 0.977 \\
$d=0.4$ & 1.068 & 1.004 & 0.996 \\
$d=0.5$ & 1.082 & 1.022 & 1.015 \\
\hline
\end{tabular}

Table 2 The standard deviation of different coupling coefficients $d$ under different noise backgrounds $(D=0.5)$

\begin{tabular}{cccc}
\hline$d / \alpha$ & $\alpha=1.5$ & $\alpha=1.8$ & $\alpha=2$ \\
\hline$d=0$ & 1.058 & 1.054 & 1.052 \\
$d=0.1$ & 1.053 & 1.034 & 1.032 \\
$d=0.2$ & 0.987 & 0.955 & 0.947 \\
$d=0.3$ & 1.003 & 0.974 & 0.968 \\
$d=0.4$ & 1.019 & 0.994 & 0.99 \\
$d=0.5$ & 1.035 & 1.014 & 1.011
\end{tabular}

It can be seen from Table 1 and Table 2 that as the intensity of noise changes, the standard deviation of the sensing system gradually changes for any system, the smaller the standard deviation, the stronger the stability of the system. According to the research in the above table, this paper chooses the coupling coefficient $d=0.2$ in order to obtain the optimal performance of the system.

\section{B. Weak signal sensing under Lévy noise}

After generating the above-mentioned Lévy noise model, take $a_{n}=2 \pi \frac{s^{n}}{s \omega f_{s}}$ as the solution step-size of the sensing system, and $A \frac{\sigma_{s d}}{\sigma_{s}} \cos \left(\omega_{1} t\right)+\eta(t)$ as the input signal, $\eta(t)$ is the generated Lévy noise. The specific steps of the intermittent chaotic signal sensing system based on the scale transformation variable step size dual-coupled Duffing oscillator established in this paper are as follows:

1. Use the scale transformation method to pretreat the signal.

2. Adjust the parameters of the intermittent chaotic signal sensing system of the variable-step dual-coupled Duffing oscillator, and set $F=0.789, \omega_{1}=1 \mathrm{rad} / \mathrm{s}$, $\alpha=1.001, d=0.2$.

3. The processed signal $A \cos \left(\omega_{1} \frac{\sigma_{s d}}{\sigma_{s}} t\right)+\eta(t) \mathrm{s}$ input into the sensing system as the input signal, the frequency is set to $1 \mathrm{kHz}$, and the initial solution step is set to $a_{n}=2 \pi \frac{s^{n}}{s \omega f_{s}}, n=1,2, \ldots, N$.

4. Adjust the solution step length and observe the $x_{1}(t)-x_{2}(t)$ timing diagram output. If the sensing system has an intermittent chaotic state between two adjacent steps $a_{n}$ and $a_{n+1}$, then indicates that the signal has been sensed, otherwise return 2 . 
5. If the signal has been sensed, the angular frequencies $\omega_{n}$ and $\omega_{n+1}$ corresponding to solution step-size are $a_{n}$ and $a_{n+1}$ can be calculated by $\omega_{n}=s^{n} \mathrm{rad} / \mathrm{s}$, then the angular frequency of the sensed signal $\ddot{\omega}=\frac{\omega_{n}+\omega_{n+1}}{2} \mathrm{rad} / \mathrm{s}$.

a. The range of signal amplitude sensible by different systems

The signal to be measured $f(t)=A \cos (10 t)$ is superimposed with the same Lévy noise $\eta(t)$ and then input into different systems for sensing. Figure 12, Figure 13, Figure 14 respectively show the intermittent chaotic single Duffing vibrator sensing system, the intermittent chaotic dual-coupled Duffing vibrator sensing system, and the sensing system established in this paper for signal sensing differential timing diagrams.
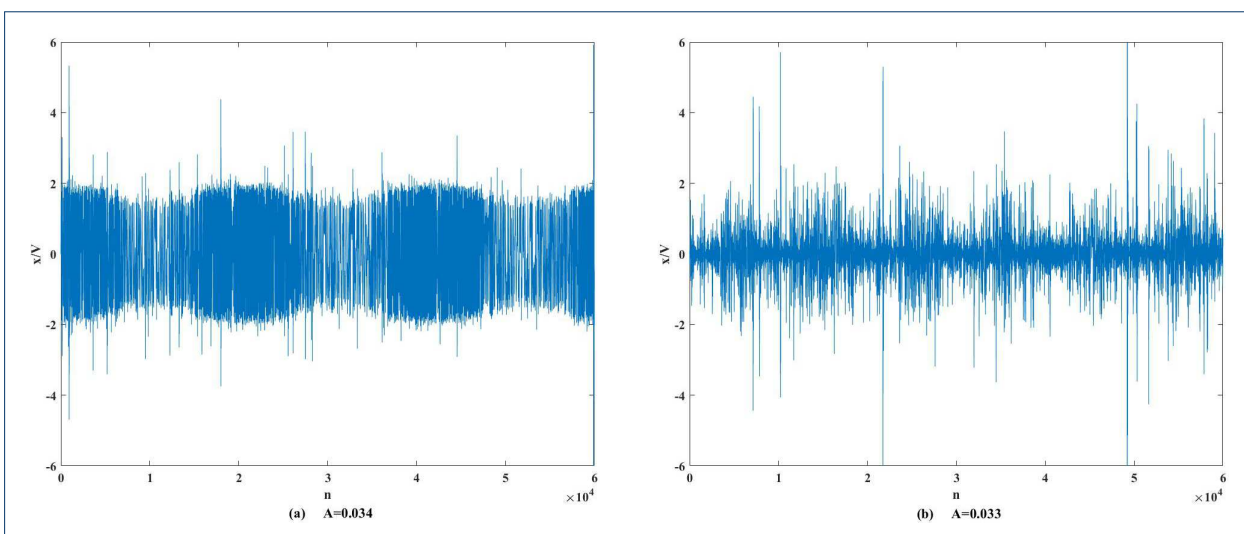

Figure 12 The intermittent chaotic single Duffing vibrator sensing system
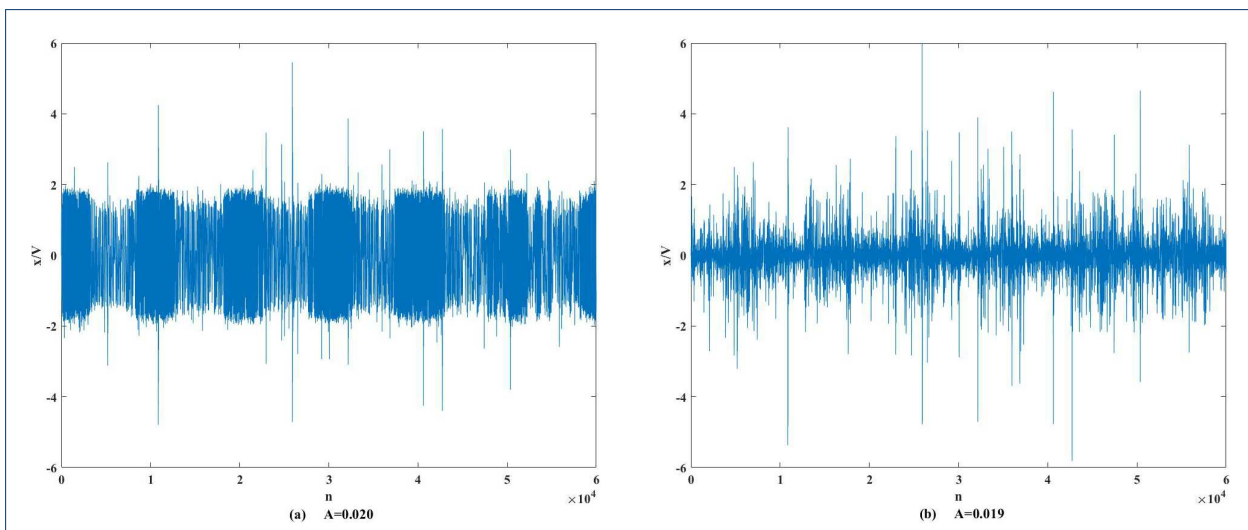

Figure 13 The intermittent chaotic dual-coupled Duffing vibrator sensing system

From Figure 12, Figure 13, Figure 14, we can see that the single Duffing oscillator and the dual-coupled Duffing oscillator have a weaker ability to sense weak signals with smaller amplitude. Our system's signal amplitude range is larger, and the sensing performance is better. 

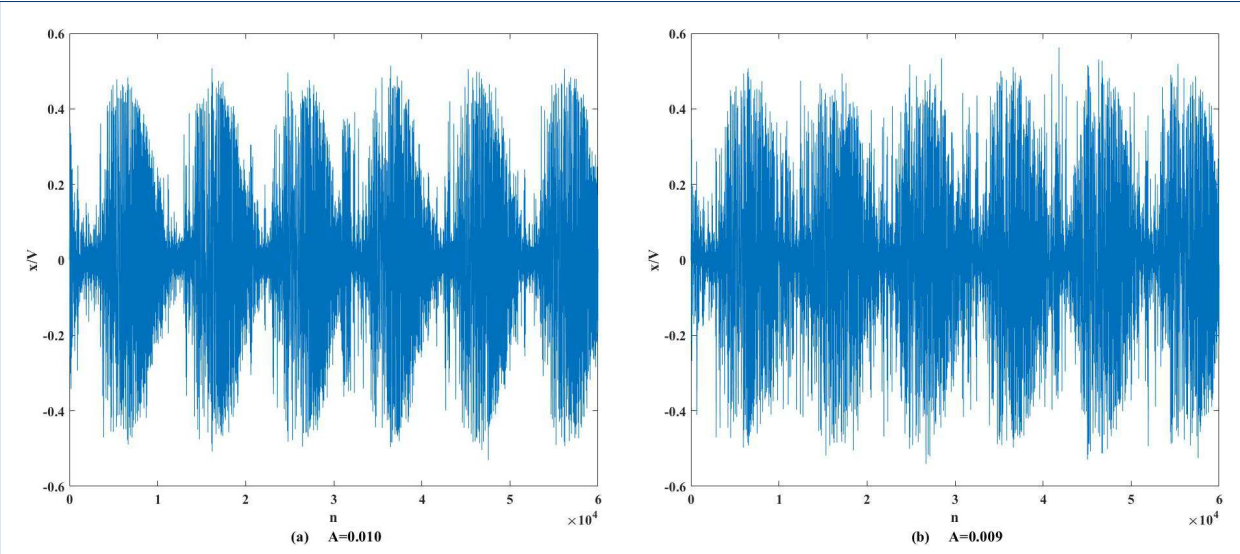

Figure 14 Our sensing system

b. Analysis of Lévy noise resistance of different systems to impacts of different intensities

The signal to be measured $f(t)=A \cos (10 t)$ is superimposed with different Lévy noise $\eta(t)$ and then input into three systems for sensing. From Figure 15(a) and Figure 15(a), it can be seen that the single Duffing vibrator and the dual-coupled Duffing vibrator have weaker sensing of weak signals under the background of strong impact Lévy noise $(\alpha=1.5,1.8)$ and have the weak noise immunity. The sensing system established in this paper can clearly sense the weak signal under the strong impact of Lévy noise $(\alpha=1.5,1.8)$, and has strong anti-interference.
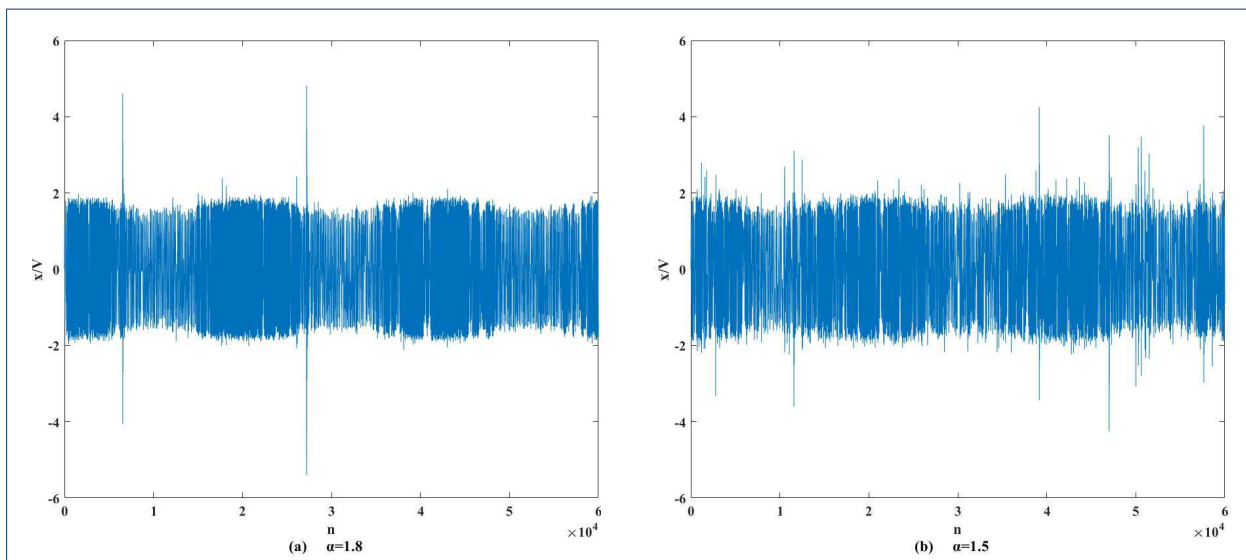

Figure 15 The intermittent chaotic single Duffing vibrator sensing system

\section{c. Analysis of system sensed SNR under the same noise background}

The signal to be measured $f(t)=A \cos (10 t)$ is superimposed with the same Lévy noise $\eta(t)$ and then input into different systems for sensing. Choose the best sensing results of the three systems. 


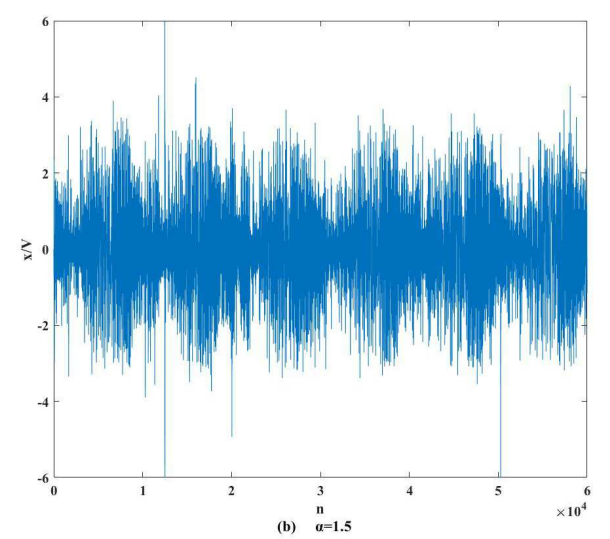

Figure 16 The intermittent chaotic dual-coupled Duffing vibrator sensing system
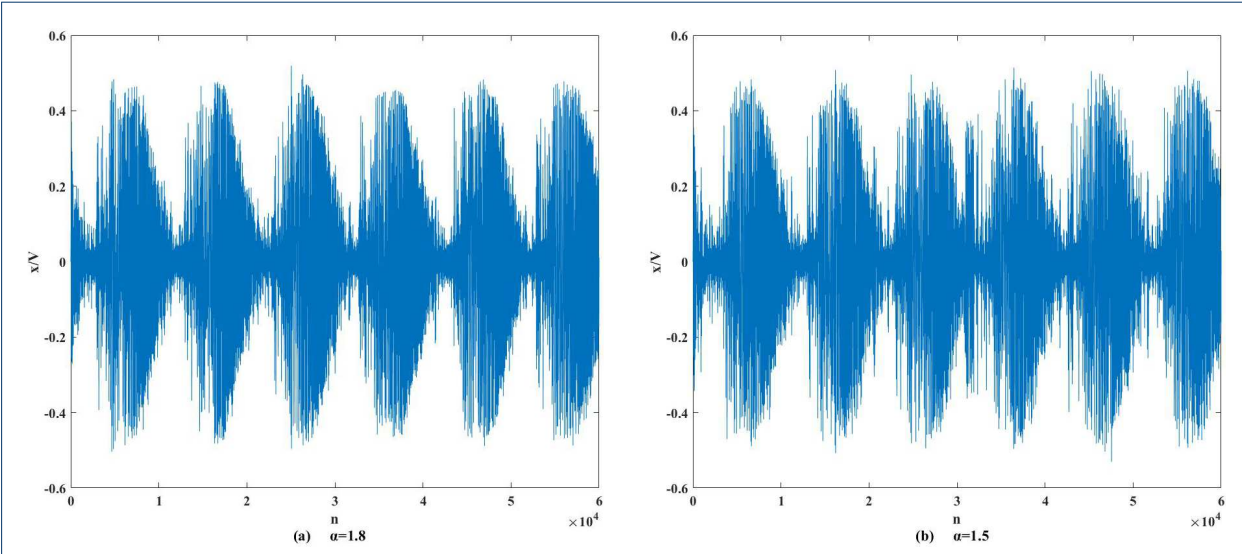

Figure 17 Our sensing system

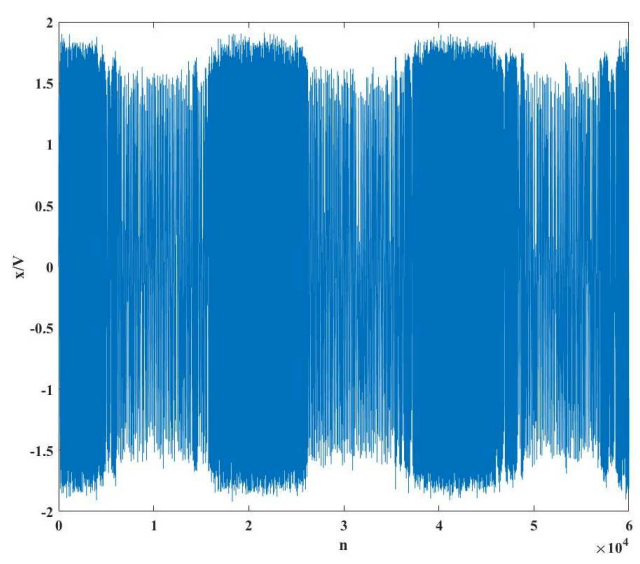

Figure 18 The intermittent chaotic single Duffing vibrator sensing system

From Figure 18, Figure 19, and Figure 20, it can be seen that when the amplitude of the signal to be measured is $\mathrm{A}$, and the solution step is $a_{39}$, all three systems will present an intermittent chaotic state, and the signal can be sensed at this time. The 
single Duffing vibrator sensing system, the dual-coupled Duffing vibrator sensing system, and the system established in this paper can respectively sense the periodic signal amplitude $A>0.034, A>0.020$ and $A>0.009$. The minimum SNR ratio of the two sensing systems can be calculated by the following formula:

$$
S N R=10 \lg \left(\frac{P_{\text {signal }}}{P_{\text {noise }}}\right)
$$

After calculation, the SNR of the single Duffing vibrator sensing system is $-32.38072162 d B$, the SNR of the dual-coupled Duffing vibrator sensing system is $-36.98970004 d B$, the SNR of the sensing system established in this paper is $-43.92544977 d B$, and the SNR ratio threshold are reduced by $11.54472815 d B$ and $6.93574973 d B$ respectively.

Since the sensing method proposed in this paper can change the common ratio to form a new solution step size and sensing bandwidth, the number of solution steps is reduced, thereby reducing the amount of calculation better. The data of each sensing system is shown in Table 3: 
Table 3 Data of each detection system

\begin{tabular}{cccc}
\hline Method & Common Ration & Number of Solving Steps & Detection Bandwidth \\
\hline \multirow{2}{*}{ Single Duffing oscillator } & 1.01 & 282 & $(1.00,1.01) \omega$ \\
& 1.02 & 197 & $(0.99,1.02) \omega$ \\
\hline \multirow{2}{*}{ Double Duffing oscillator } & 1.03 & 98 & $(0.98,1.03) \omega$ \\
\hline \multirow{2}{*}{ Ours } & 1.05 & 68 & $(0.97,1.05) \omega$ \\
& 1.06 & 60 & $(0.96,1.06) \omega$ \\
& 1.07 & 57 & $(0.96,1.07) \omega$ \\
\hline & 1.06 & 60 & $(0.95,1.07) \omega$ \\
& 1.07 & 57 & $(0.94,1.08) \omega$ \\
\hline
\end{tabular}

It can be seen from Table 3 that the number of solving steps and the amount of calculation of the sensing system established in this paper are obviously the smallest.

\section{d. Multi-frequency signal sensing performance}

In actual underwater signal sensing, multiple signals will be sensed. We change the input signal to $0.01 \cos (10 t)+0.01 \cos (20 t)$, and the Lévy noise takes $\alpha=1.5$ respectively $(\eta(t))$, The sensing results are shown in Figure 21 and Figure 22:
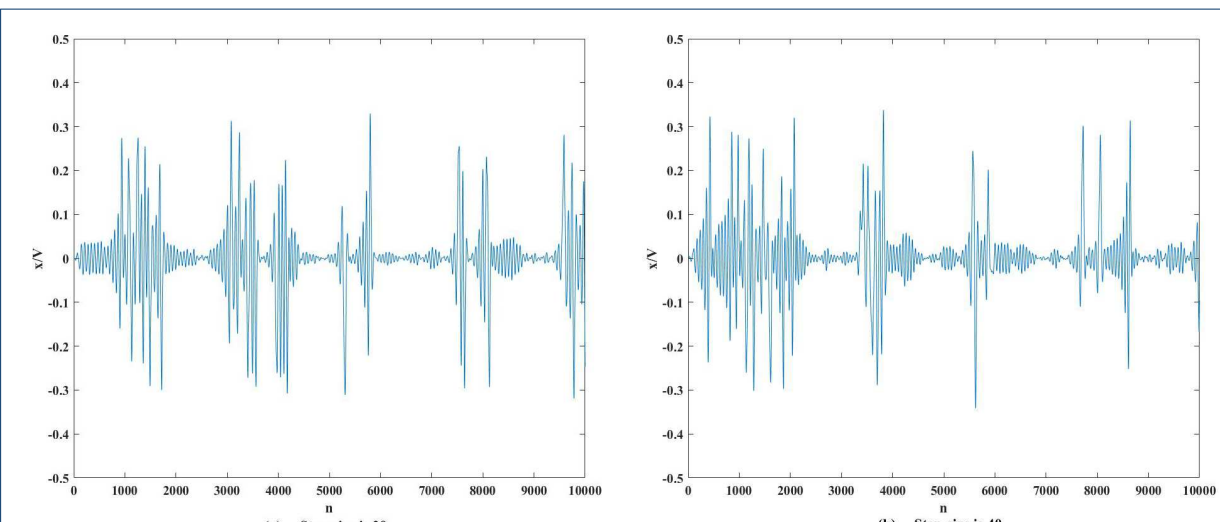

(a) Step-size is 39

(b) Step-size is 40

Figure 21 The sensing result of the first segment of the multi-frequency signal

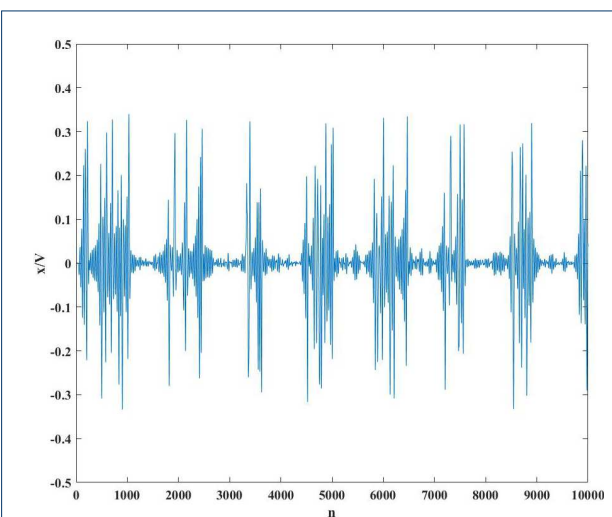

(a) Step-size is 51

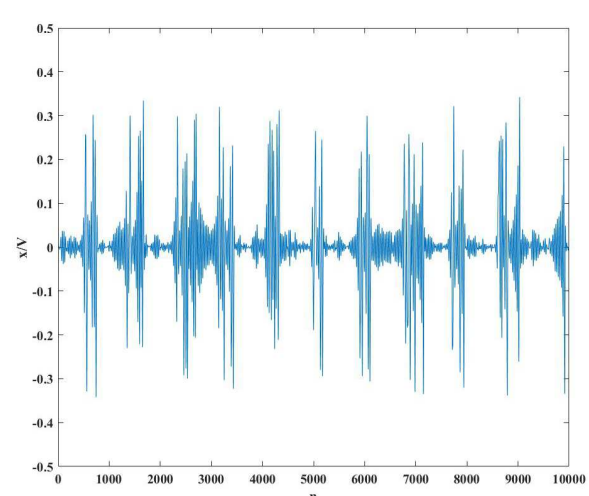

(b) Step-size is 52

Figure 22 The sensing result of the second segment of the multi-frequency signal 
From Figure 21 and Figure 22, the sensing system established in this paper can show intermittent chaos when sensing two signals of different frequencies. The signals of the two frequencies are sensitive, so it cannot be determined that the signal can be sensed. Therefore, the method proposed in this paper has obvious advantages in sensing unknown signals in multiple frequency bands.

When the solution step-size are $a_{39}$ and $a_{40}$, the corresponding sizes of $f_{39}$ and $f_{40}$ are $9.87 \mathrm{~Hz}$ and $10.08 \mathrm{~Hz}$ respectively, and then the judgment frequency of the sensing system established in this paper is $f_{10}=10 \mathrm{~Hz}$. When the solution step-size are $a_{51}$ and $a_{52}$, the corresponding sizes of $f_{51}$ and $f_{52}$ are $19.77 \mathrm{~Hz}$ and $20.10 \mathrm{~Hz}$ respectively, and the sensing system judges the frequency of the signal is $f_{20}=20 \mathrm{~Hz}$. When frequencies are $10 \mathrm{~Hz}$ and $20 \mathrm{~Hz}$, the calculation error rate is less than $0.03 \%$, that is, it can obtain the frequency of the signal to be sensed more accurately under the interference of a strong noise environment.

\section{e. Actual underwater acoustic data detection}

Figure 23 shows the low-frequency underwater data collected by a reservoir in Sichuan Province, China. In order to facilitate the analysis, the actual underwater acoustic data was sampled again at a sampling frequency of $1 \mathrm{kHz}$. After obtaining the underwater acoustic signal data, the normalization operation was carried out and then sent to the sensing system based on this paper. From Figure 23, it can be found that there is strong impulsive noise underwater and strong broadband interference noise at many moments, which is more consistent with the Lévy noise generated by simulation, which shows that it is reasonable and appropriate to use Lévy noise to describe underwater environmental noise.
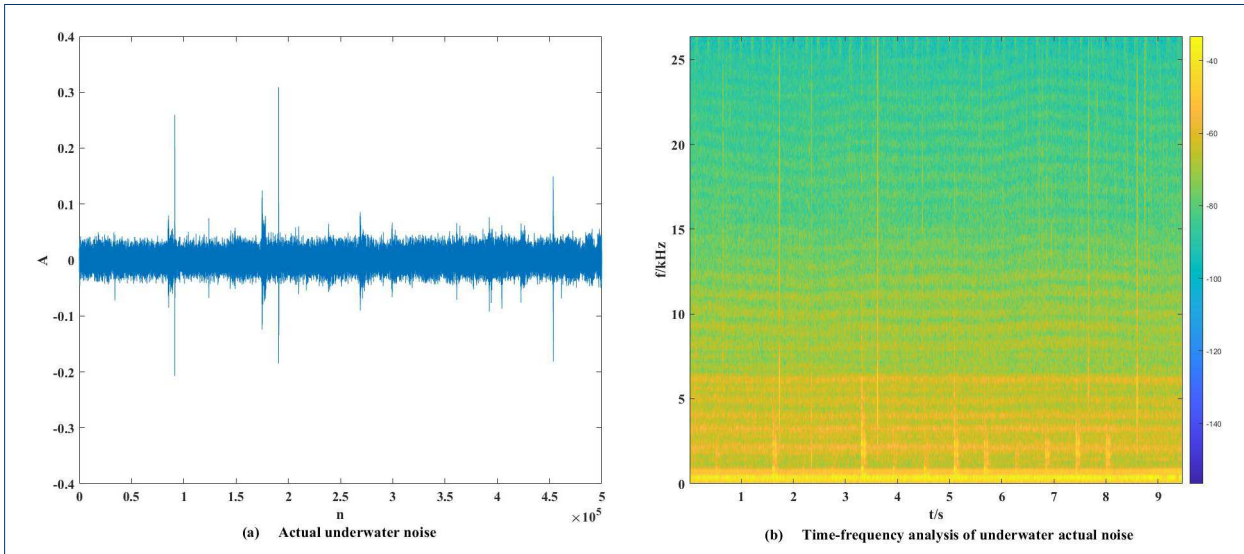

Figure 23 Actual underwater acoustic data

Figure 24 is the power spectral density diagram of the signal. It can be observed that the approximate signal frequency is about $10 \mathrm{~Hz}$. In order to sense the actual signal, we set the initial solution step-size is $a_{n}=2 \pi \frac{s^{n}}{s \omega f_{s}}, s=1.06$ and input the actual signal into the sensing system established in this paper to obtain the result as shown in Figure 25:

In Figure 25, the step-size of the sensing system are $a_{39}$ and $a_{40}$, it shows an obvious intermittent chaotic state. The corresponding magnitudes of $f_{39}$ and $f_{40}$ 
are $9.87 \mathrm{~Hz}$ and $10.08 \mathrm{~Hz}$, respectively, and the system judges that the frequency of the signal which to be sensed at this time is $f_{10}=9.99 \mathrm{~Hz}$. When the frequency of the signal to be measured is $10 \mathrm{~Hz}$, the calculation error rate is lee than $0.02 \%$. Our sensing system is more accurately under the interference of a strong natural environment. It verifies the effectiveness and scientificity of the sensing system established in this paper in practical applications.

\section{Conclusions}

This paper has proposed a Lévy noise model to describe the underwater natural environment under substantial interference for the underwater information sensing task. It describes the actual underwater environment more accurately and provides an analysis and estimation method to select better parameters; Aiming at the sensing problem of unknown frequency signal under the Lévy noise, this paper has proposed an improved dual-coupled Duffing oscillator signal sensing method based on the variable step-size method and scale transformation. The method has better resistance to intense noise, better adaptability to all kinds of impact noise, higher sensing efficiency, more intuitive sensing results, and lower SNR ratio threshold; Under the interference of Lévy noise, the improved dual-coupled Duffing oscillator signal sensing system based on variable step size intermittent chaos has established. A large number of simulation experiments show the effectiveness and superiority 
of the system. The lowest SNR threshold is $-43.92544977 \mathrm{~dB}$. The error rate of frequency estimation is less than $0.02 \%$; Finally, experiments are carried out with actual underwater acoustic data to verify the effectiveness and scientificity of the sensor system established in this paper in practical application.

\author{
Acknowledgements \\ We gratefully acknowledge the people who gave meticulous and valuable comments on this paper and the \\ anonymous reviewers who spent the valuable time in reviewing our paper. \\ Funding \\ This work was supported in part by the National Natural Science Foundation of China (No.62072074, No.62076054, \\ No.62027827, No.61902054, No.62002047), the Frontier Science and Technology Innovation Projects of National \\ Key R\&D Program (No.2019QY1405), the Sichuan Science and Technology Innovation Platform and Talent Plan \\ (No.2020JDJQ0020), the Sichuan Science and Technology Support Plan (No.2020YFSY0010).
}

Availability of data and materials

The datasets generated or analyzed during the current study are not publicly available due [REASON WHY DATA ARE NOT PUBLIC] but are available from the corresponding author on reasonable request.

Competing interests

The authors declare that they have no competing interests.

Consent for publication

All authors have agree and given their consent for submission of this paper to Euraship Journal of Wireless Communication.

Authors' information

Not applicable.

Authors' contributions

All authors reviewed and edited the manuscript. All authors read and approved the final manuscript.

Abbreviations

Not applicable.

Author details

${ }^{1}$ School of Automation Engineering, University of Electronic Science and Technology of China, Chengdu, China. ${ }^{2}$ School of Physical Science and Technology, Southwest Jiaotong University, Chengdu, China. ${ }^{3}$ Network and Data Security Key Laboratory of Sichuan Province, Chengdu, China.

References

1. Chen, D., Zhao, Z., Qin, X., Luo, Y., Cao, M., Xu, H., Liu, A.: Magleak: A learning-based side-channel attack for password recognition with multiple sensors in iiot environment. IEEE Transactions on Industrial Informatics (2020)

2. Zhang, N., Yang, P., Zhang, S., Chen, D., Zhuang, W., Liang, B., Shen, X.S.: Software defined networking enabled wireless network virtualization: Challenges and solutions. IEEE Network 31(5), $42-49$ (2017). doi:10.1109/MNET.2017.1600248

3. Zhang, N., Yang, P., Ren, J., Chen, D., Yu, L., Shen, X.: Synergy of big data and $5 \mathrm{~g}$ wireless networks: Opportunities, approaches, and challenges. IEEE Wireless Communications 25(1), 12-18 (2018). doi:10.1109/MWC.2018.1700193

4. Ale, L., Zhang, N., Wu, H., Chen, D., Han, T.: Online proactive caching in mobile edge computing using bidirectional deep recurrent neural network. IEEE Internet of Things Journal 6(3), 5520-5530 (2019)

5. Ding, Y., Wu, G., Chen, D., Zhang, N., Gong, L., Cao, M., Qin, Z.: Deepedn: a deep-learning-based image encryption and decryption network for internet of medical things. IEEE Internet of Things Journal 8(3), 1504-1518 (2020)

6. Tian, T., Liu, G., Sun, D.: Sonar Technology. Harbin. Harbin Engineering University Press (2006)

7. Liu, X., Qin, y.: Modem marine power vs state marine strategy. Journal of Social Sciences, 73-79 (2004)

8. Peyvandi, H., Farrokhrooz, M., Roufarshbaf, H., Park, S.-J.: Sonar systems and underwater signal processing: classic and modern approaches. SONAR systems, 173-206 (2011)

9. Ma, S., Wang, H., Shen, X., Dong, H.: Stochastic resonance for underwater vlf weak signal detection under lévy noise background. In: 2017 IEEE International Conference on Signal Processing, Communications and Computing (ICSPCC), pp. 1-5 (2017). IEEE

10. Biao, W., Yu, F., Yang, W., He, C.: An adaptive data detection algorithm based on intermittent chaos with strong noise background. Neural Computing and Applications 32(22), 16755-16762 (2020)

11. Chi, C., Pallayil, V., Chitre, M.: Design of an adaptive noise canceller for improving performance of an autonomous underwater vehicle-towed linear array. Ocean Engineering 202, 106886 (2020)

12. Andrew, R.K., Howe, B.M., Mercer, J.A., Dzieciuch, M.A.: Ocean ambient sound: comparing the 1960s with the 1990s for a receiver off the california coast. Acoustics Research Letters Online 3(2), 65-70 (2002)

13. Park, J.S., Manocha, D.: Efficient probabilistic collision detection for non-gaussian noise distributions. IEEE Robotics and Automation Letters 5(2), 1024-1031 (2020) 
14. John, A., Sadasivan, J., Seelamantula, C.S.: Adaptive savitzky-golay filtering in non-gaussian noise. IEEE Transactions on Signal Processing 69, 5021-5036 (2021)

15. Korakas, A., Hovem, J.M.: Comparison of modeling approaches to low-frequency noise propagation in the ocean. In: 2013 MTS/IEEE OCEANS-Bergen, pp. 1-7 (2013). IEEE

16. Haxel, J.H., Dziak, R.P., Matsumoto, H.: Observations of shallow water marine ambient sound: The low frequency underwater soundscape of the central oregon coast. The Journal of the Acoustical Society of America 133(5), 2586-2596 (2013)

17. Siderius, M., Gebbie, J.: Environmental information content of ocean ambient noise. The Journal of the Acoustical Society of America 146(3), 1824-1833 (2019)

18. Yanga, M.: Fractional stochastic differential equations driven by levy noise. Filomat 35(7), $2403-2424$ (2021)

19. Beltaief, S., Chernoyarov, O., Pergamenchtchikov, S.: Model selection for the robust efficient signal processing observed with small levy noise. Annals of the Institute of Statistical Mathematics 72(5), 1205-1235 (2020)

20. Wang, J., Li, J., Yan, S., Shi, W., Yang, X., Guo, Y., Gulliver, T.A.: A novel underwater acoustic signal denoising algorithm for gaussian/non-gaussian impulsive noise. IEEE Transactions on Vehicular Technology 70(1), 429-445 (2020)

21. Liu, S., Sun, Y., Kang, Y.: A novel e-exponential stochastic resonance model and weak signal detection method for steel wire rope. IEEE Transactions on Industrial Electronics (2021)

22. Jing, S., Hall, J., Zheng, Y.R., Xiao, C.: Signal detection for underwater iot devices with long and sparse channels. IEEE Internet of Things Journal 7(8), 6664-6675 (2020)

23. Moatimid, G.M.: Stability analysis of a parametric duffing oscillator. Journal of Engineering Mechanics 146(5), 05020001 (2020)

24. Liu, M., Chen, J., Jiang, H., Yu, Z., Hu, C., Lu, B.: Synchronization of chaotic delayed systems via intermittent control and its adaptive strategy. Nonlinear Analysis: Modelling and Control 26(6), 993-1011 (2021)

25. Li, N., Li, X., Liu, C.: Detection method of a short-time duffing oscillator array with variable amplitude coefficients. Journal of Harbin Engineering University 37(12), 1645-1652 (2016)

26. Zhou, S., Lin, C.: Application of chaos theory for weak signal of ship detecting. J. Wuhan Univ. Technol. 33(1), 161-164 (2009)

27. $\mathrm{Li}, \mathrm{S} ., \mathrm{Wu}, \mathrm{X}$.: Application of ale based on $\mathrm{ftf}$ algorithm in ship-radiated noise detection. Communications Technology 50(06), 1175-1180 (2017)

28. Sun, Q., Zhang, J.: Weak signal detection based on improved chaotic oscillator system with dual coupling. Comput. Mod. (1), 17-21 (2012)

29. Li, G., Hou, Y., Yang, H.: A new duffing detection method for underwater weak target signal. Alexandria Engineering Journal (2021)

30. Brzeźniak, Z., Liu, W., Zhu, J.: The stochastic strichartz estimates and stochastic nonlinear schrödinger equations driven by levy noise. Journal of Functional Analysis 281(4), 109021 (2021)

31. Xu, W., Hao, M., Gu, X., Yang, G.: Stochastic resonance induced by levy noise in a tumor growth model with periodic treatment. Modern Physics Letters B 28(11), 1450085 (2014)

32. Ma, S.: Research on very low frequency seismic wave detection technology based on stochastic resonance under levi noise. Master's thesis, Northwestern Polytechnical University, Xian, Xian (2018)

33. $\mathrm{Ma}, \mathrm{X}$., Nikias, C.L.: Parameter estimation and blind channel identification in impulsive signal environments. IEEE Transactions on signal processing 43(12), 2884-2897 (1995)

34. Deng, L., Zhao, X., Yin, B.: A method of extracting underwater acoustic beaconing signal. In: 2021 OES China Ocean Acoustics (COA), pp. 744-747 (2021). IEEE

35. Li, G., Hou, Y., Yang, H.: A new duffing detection method for underwater weak target signal. Alexandria Engineering Journal (2021)

36. Li, G., Zhao, K., Yang, H.: A new method for detecting line spectrum of ship-radiated noise based on a new double duffing oscillator differential system (2020)

37. Wang, K., Yan, X., Yang, Q., Hao, X., Wang, J.: Weak signal detection based on strongly coupled duffing-van der pol oscillator and long short-term memory. Journal of the Physical Society of Japan 89(1), 014003 (2020)

38. Gu, X., Lou, J., Liu, K., Hu, P.: Underwater detection signal based on Im-bp neural network algorithm. In: Journal of Physics: Conference Series, vol. 1533, p. 032035 (2020). IOP Publishing

\section{Additional Files}

Figure Title and Legend Section

Figure 1 - Time-domain distribution map of live noise corresponding to different feature index $\alpha$.

Figure 2 - The particle probability distribution of the system output under the excitation of different noise intensity $D$.

Figure 3 - Estimation of characteristic index $\alpha$

Figure 4 - Estimation of noise intensity $D$.

Figure 5 - The output sequence of the single Duffing oscillator system.

Figure 6 - The output sequence of the dual-coupled Duffing oscillator system.

Figure 7 - The output sequence of improved method.

Figure 8 - The specific steps of the chaotic sensing system.

Figure 9 - The the wrong chaotic state of the chaotic sensing system.

Figure 10 - The scale transformation flow chart.

Figure 11 - Intermittent chaos detection system with scale conversion.

Figure 12 - The intermittent chaotic single Duffing vibrator sensing system.

Figure 13 - The intermittent chaotic dual-coupled Duffing vibrator sensing system.

Figure 14 - Our sensing system.

Figure 15 - The intermittent chaotic single Duffing vibrator sensing system

Figure 16 - The intermittent chaotic dual-coupled Duffing vibrator sensing system.

Figure 17 - Our sensing system. 
Figure 18 - The intermittent chaotic single Duffing vibrator sensing system.

Figure 19 - The intermittent chaotic dual-coupled Duffing vibrator sensing system.

Figure 20 - Our sensing system.

Figure 21 - The sensing result of the first segment of the multi-frequency signal.

Figure 22 - The sensing result of the second segment of the multi-frequency signal.

Figure 23 - Actual underwater acoustic data.

Figure 24 - Power spectral density diagram.

Figure 25 - The sensing result of the actual underwater acoustic data. 


\section{Supplementary Files}

This is a list of supplementary files associated with this preprint. Click to download.

- bic.bib

- bmcmathphys.bst

- bmcartbiblio.sty

- bmcart.cls

- spbasic.bst

- vancouver.bst 